\title{
ESTUDO DA CINÉTICA E EQUILÍBRIO DA ADSORÇÃO DA ÁGUA PRODUZIDA DO PETRÓLEO NA REMOÇÃ̃O DE CARBONO ORGÂNICO
}

\author{
P. C. A. P de Carvalho ${ }^{1}$, O. Chiavone - Filho $^{1}$, J. M. M. Quinino ${ }^{1}$, L. A. S. Saldanha ${ }^{1}$, N. \\ S.Figueiredo ${ }^{1}$ \\ ${ }^{1}$ Universidade Federal do Rio Grande do Norte, Departamento de Engenharia Química ${ }^{1}$ \\ E-mail para contato: pcpuglia@hotmail.com
}

RESUMO - A água produzida constitui um problema ambiental, uma vez que apresenta composição química tóxica, alguns desses componentes são os orgânicos dispersos e dissolvidos. Diversos são os tratamentos existentes que visam à remoção dos orgânicos dissolvidos, que por sua vez não podem ser retirados por tratamentos convencionais de separação por gravidade. A adsorção vem sendo estudada e vários são os adsorventes existentes no mercado, sendo um deles o carvão ativado, que já provou ser um excelente adsorvente. No trabalho foram determinados os modelos cinéticos de adsorção para o efluente sintético $\left(25^{\circ} \mathrm{C}, 37^{\circ} \mathrm{C}\right.$ e $\left.50^{\circ} \mathrm{C}\right)$ e a água produzida real $\left(25^{\circ} \mathrm{C}\right)$, também foram obtidos os parâmetros das isotermas de Langmuir e Freundlich para o efluente sintético $\left(25^{\circ} \mathrm{C}\right)$ em carvão ativado de casca de coco (8 - 12 mesh). Os resultados mostraram que tanto para o efluente sintético como para a água produzida o melhor modelo cinético, que expressa à adsorção é o pseudo - segunda ordem. A cinética é favorecida na faixa de temperatura de 25 a $37^{\circ} \mathrm{C}$, a redução na adsorção ocorreu na temperatura de $50^{\circ} \mathrm{C}$. Dentre os modelos testados o modelo de Langmuir e Freundlich, apresentaram bons ajustes (0,988 Langmuir e 0,955 Freundlich), prevalecendo então o modelo de Langmuir.

\section{INTRODUÇÃO}

A água de produção ou água produzida ocorre comumente durante a produção de gás e óleo, nas plataformas de operação offshore e onshore. A água produzida consiste na água de formação, que ocorre naturalmente nos reservatórios ou de água de injeção ou ainda da mistura de ambas (Veil et al, 2004). A água produzida possui uma variedade de produtos químicos, que têm sido dissolvidos na formação durante milhares de anos. Estes incluem sais inorgânicos, vários metais e uma diversidade de compostos orgânicos e elementos radioativos naturais, que podem ser encontrados em concentrações traços (Neff et al, 1992). A presença dos componentes químicos caracteriza a água produzida como sendo uma fonte agressora ao meio ambiente, um exemplo é a variedade de compostos orgânicos dispersos e dissolvidos presentes que apresentam grande risco ao meio ambiente. Sendo assim sua remoção é um fator de grande relevância, tendo em vista que alguns desses compostos orgânicos podem promover intoxicações agudas e/ou crônicas em organismos aquáticos (Mariano, 2005). O tratamento dos efluentes da indústria petrolífera é uma 


\section{9 a 22 de outubro de 2014 \\ Florianópolis/SC}

prática extremamente importante, principalmente se o destino final for o descarte no meio ambiente, o alto volume gerado, é a sua composição complexa são fatores relevantes (Vegueria et al, 2002).

Diversos são os tratamentos existentes, têm-se os processos de natureza física, química, biologia e físico - química. Cada processo terá suas limitações e irá depender das substâncias contidas no efluente, se estão dissolvidas ou dispersas. A grande vantagem da adsorção quando comparados a outros processos de separação, está no fato deste possuir uma elevada seletividade molecular, permitindo a separação de vários componentes com um baixo consumo energético (Rutvhen, 1984). Outra vantagem do uso da adsorção para eliminação dos contaminantes em relação a outras tecnologias, esta na possibilidade de recuperação do produto para reutilizá-lo e a alta eficiência de remoção com baixa concentração inicial (Benkhedda et al, 2000). A adsorção é definida como uma operação de transferência de massa do tipo sólido - fluido, no qual se explora a habilidade de certos sólidos em concentrar na superfície determinadas substâncias existentes em substâncias líquidas ou gasosas, permitindo separá-las dos demais componentes da solução (Gomide, 1980). A adsorção em carvão ativado para eliminação de contaminantes orgânicos é um processo bem conhecido, um grande número de publicações técnicas cientificas aborda a remoção de orgânicos; adsorção de benzeno e tolueno (Lilo - Ródenas et al, 2006), adsorção de fenol (Roostaei e Tezel, 2003; Laszló, Podkoscielny e Dabrowsk, 2005), são alguns exemplos. Muitos trabalhos de adsorção têm sido voltados para o estudo da cinética de adsorção alguns exemplos são: cinética de adsorção de fenol sobre carvão ativado (Lorenc - Grabowska et al, 2013; Alhamed, 2009), cinética da adsorção de tolueno sobre carvão (Pei e Zhang, 2012). A cinética de adsorção descreve a velocidade com o qual as moléculas do adsorbato são adsorvidas pelo adsorvente. A velocidade de adsorção depende das características físico - química do adsorbato (natureza, peso molecular, solubilidade etc.), do adsorvente (natureza estrutura dos poros) e da solução (temperatura e pH) (Claudino, 2003). O mecanismo de adsorção pode ser descrito, por três etapas consecutivas são elas: transferência de massa externa do adsorvato em solução para a superfície do adsorvente, difusão do adsorvato para os sítios de adsorção no interior do adsorvente e na últimaetapa ocorre a difusão propriamente dita. As etapas de transferência de massa externa e de difusão do adsorvato são as determinantes na velocidade de adsorção, enquanto que a última etapa não oferece nenhuma resistência ao processo (McKAY, 1996).

Outro importante ponto de estudo da adsorção são os modelos de isoterma de adsorção, os quais têm como alvo descrever a concentração de adsorvato no adsorvente no equilíbrio, em função da concentração de adsorvato na fase líquida em contato com o adsorvente também no equilíbrio. O objetivo deste trabalho foi estudar a cinética e o equilíbrio de adsorção da água produzida do petróleo, focando a remoção da carga orgânica sobre o carvão ativado.

\section{METODOLOGIA}

\subsection{Cinética e equilíbrio de adsorção}

Para determinação da cinética os experimentos foram conduzidos em batelada, em erlenmeyers de $250 \mathrm{~mL}$, contendo cada frasco $100 \mathrm{~mL}$ de água produzida sintética e $5 \mathrm{~g}$ de carvão ativado de coco de granulometrias (8 -12 mesh), sob agitação constante de $120 \mathrm{rpm}$ em uma incubadora digital e temperaturas de $25^{\circ} \mathrm{C}, 37^{\circ} \mathrm{C}$ e $50^{\circ} \mathrm{C}$ e $\mathrm{pH}$ neutro. $\mathrm{O}$ efluente sintético foi feito a partir de uma mistura 
de orgânicos; xileno (10 mg. $\left.\mathrm{L}^{-1}\right)$, N-heptano $\left(10 \mathrm{mg} \cdot \mathrm{L}^{-1}\right)$ e fenol $\left(200 \mathrm{mg} . \mathrm{L}^{-1}\right)$ de modo a obter uma carga orgânica em torno de $140 \mathrm{mg} . \mathrm{L}^{-1}$ de TOC e cloreto de sódio (1000 mg. $\left.\mathrm{L}^{-1}\right)$. A água produzida real foi proveniente de uma Estação de Tratamento de Efluente de uma Unidade Operacional RN-CE (UO - RNCE). Os experimentos comparativos dos dois efluentes, sintético e real foram realizados com pH neutro e temperatura de $25^{\circ} \mathrm{C}$. Em intervalos de tempo pré-determinado foram removidas alíquotas de $40 \mathrm{ml}$ para determinação da cinética, as concentração de carbono orgânico total (TOC $\mathrm{mg} / \mathrm{L}$ ) das amostras foram determinadas através do total organic analyzer COT - Vcph da shimadzu. Os experimentos de equilíbrio de adsorção foram feitos de forma semelhante à cinética, diferindo apenas no tempo de agitação, que foi de 30 minutos e a temperatura fixa de $25^{\circ} \mathrm{C}$, somente para $\mathrm{o}$ efluente sintético.

\section{RESULTADOS E DISCUSSÃO}

\subsection{Cinética de adsorção}

A Figura 3.1 ilustra a variação da quantidade adsorvida (q) de carbono orgânico total (TOC, mg. $\mathrm{L}^{-1}$ ) do efluente em função do tempo de contato com o adsorvente, em três temperaturas diferentes $\left(25^{\circ} \mathrm{C}, 37^{\circ} \mathrm{C}\right.$ e $\left.50^{\circ} \mathrm{C}\right)$. Foi observado que para ambas as temperaturas o equilíbrio ocorreu após 25 minutos de experimento. Também foi verificado que a faixa de temperatura de $25^{\circ} \mathrm{C}$ a $37^{\circ} \mathrm{C}$ apresentou uma melhor adsorção, quando comparada à temperatura de $50^{\circ} \mathrm{C}$.

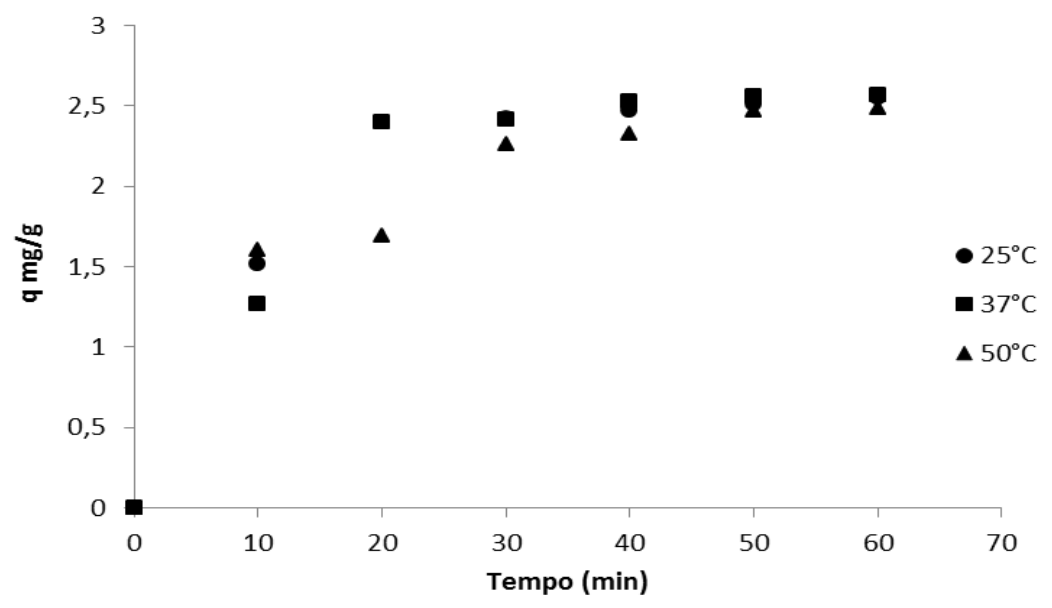

Figura 3.1 - Cinética de adsorção em diferentes temperaturas para o efluente sintético

Maretto et al, 2014 avaliou a eficácia do uso da sílica na adsorção de poluentes orgânicos (Tolueno e benzeno), mostrando uma remoção de $80 \%$, com uma cinética de adsorção de algumas horas para altas concentrações. Para estudar o mecanismo que controla o processo de adsorção, tais como, reação química e transferência de massa na solução, dois modelos cinéticos foram testados para interpreter os dados experimentais. Uma boa correlação dos dados cinéticos revelou o mecanismo de adsorção, sendo assim o coeficiente angular de cada equação cinética foi escolhido para interpreter o mecanismo de adsorção da carga orgânica do efluente sintético em 
diferentes temperaturas e para a água produzida real. A Cinética de adsorção da carga orgânica do efluente sintético e real foi analisada a partir dos modelos cinéticos de pseudo primeira ordem (Figura3. 2) e pseudo - segunda ordem (3.3), no qual foram propostos por Lagergreen em 1898. O modelo pseudo primeira ordem é expresso de acordo com a Equação (3.1).

$$
\frac{d_{q e}}{d t}=k_{1}\left(q_{e}-q_{t}\right)
$$

Onde $q_{e}$ e $q_{t}$ em (mg. $g^{-1}$ ) são a capacidade de adsorção no equilíbrio e no tempo $t$, respectivamente e $\mathrm{k}_{1}\left(\mathrm{~h}^{-1}\right)$ e a constante de Lagergreen da cinética pseudo - primeira ordem. Integrando a Equação (3.1) nos limites, $\mathrm{t}=0$ a $\mathrm{t}=\mathrm{t}$ e $\mathrm{q}_{\mathrm{t}}=0$ a $\mathrm{q}_{\mathrm{t}}=\mathrm{q}_{\mathrm{e}}$, temos a Equação (3.2).

$$
\log \left(q_{e}-q_{t}\right)=\log q_{e}-\frac{k_{1}}{2,303} t
$$

O modelo de pseudo- segunda ordem pode ser expresso pela Equação (3.3):

$$
\frac{d q_{e}}{d t}=k_{2}\left(q_{B}-q_{t}\right)^{2}
$$

Em que $\mathrm{k}_{2}$ é a constant de velocidade de segunda ordem. Fazendo a integração da Equação (3.4) e aplicando as condições iniciais, tem-se:

$$
\frac{t}{q_{e}}=\frac{1}{k_{2} q_{\theta}^{2}}+\frac{1}{q_{e}} t
$$

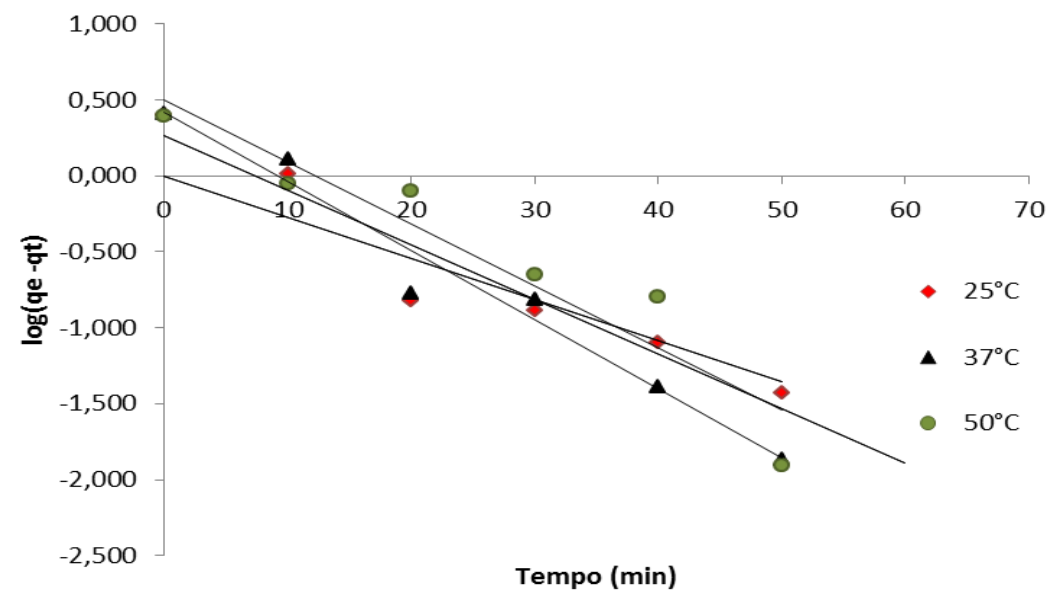

Figura 3.2 - Modelo pseudo - primeira ordem da adsorção da carga orgânica do efluente sintético 


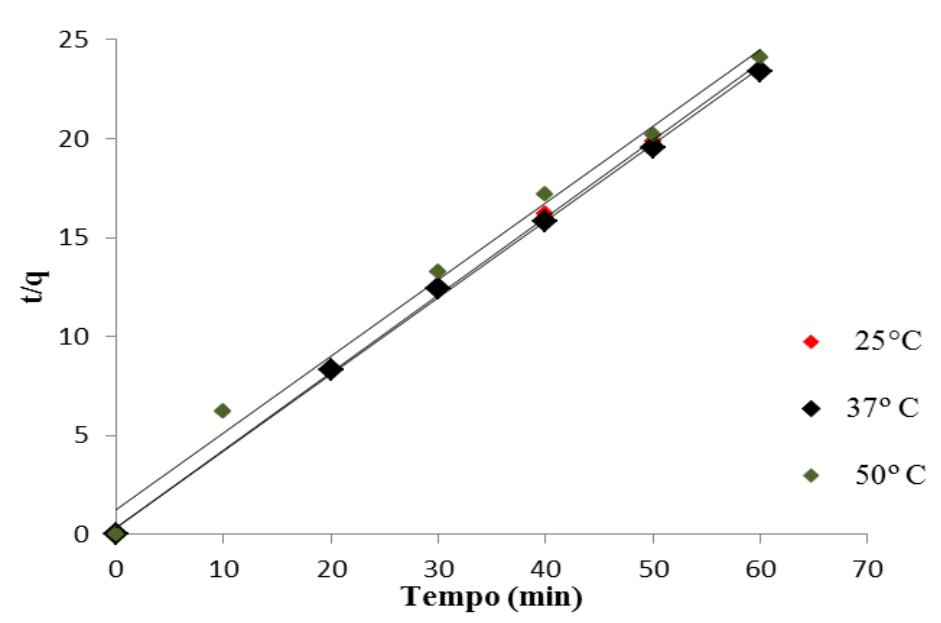

Figura 3.3 - Modelo pseudo - segunda ordem da adsorção da carga orgânica do efluente sintético

Os dois modelos apresentaram boas correlações para toda faixa de temperatura estudada, porém o modelo de pseudo segunda ordem revelou melhores ajustes, indicando ser o modelo que melhor explica a adsorção. Os parâmetros das regressões lineares determinados empregando os modelos cinéticos estão apresentados nas Tabelas 3.1 e 3.2. Os estudos cinéticos revelaram que a equação de pseudo segunda ordem forneceu os melhores ajustes dos dados experimentais. De acordo com Ho e Mackay (2000) e Ho (2006) este modelo descreve bem o processos de adsorção química, envolvendo a troca ou a doação de elétrons entre o adsorvato e o adsorvente, como forças covalentes e de troca iônica. No entanto Ho e Mackay (1998) sugerem que quando ambos os parâmetros (qe e h) diminuem com o aumento da temperatura, pode estar ocorrendo à adsorção química e física. Sendo assim, há uma hipótese que a adsorção do carbono orgânico total, está sendo conduzida pelos dois mecanismos.

Tabela 3.1 - Parâmetros da regressão linear e as constants de velocidade determinado usando os modelos cinéticos de pseudo - primeira ordem

\begin{tabular}{c|c|c|c}
\hline $\begin{array}{c}\text { Temperatura } \\
\left({ }^{\circ} \mathbf{C}\right)\end{array}$ & Equação & $\mathbf{R}$ & $\mathbf{K}$ \\
\hline 25 & $\mathrm{y}=-0,0359 \mathrm{x}+0,2631$ & 0,9607 & $-0,0334$ \\
37 & $\mathrm{y}=-0,0455 \mathrm{x}+0,4192$ & 0,9839 & $-0,048$ \\
50 & $\mathrm{Y}=-0,0408 \mathrm{X}+0,5023$ & 0,9476 & $-0,111$ \\
& & & \\
\hline
\end{tabular}


Tabela 3.2 - Parâmetros dos modelos de pseudo- segunda ordem

\begin{tabular}{c|c|c|c|c|c}
\hline Temperatura $\left({ }^{\circ} \mathrm{C}\right)$ & $\begin{array}{c}\mathrm{qe} \\
\left(\mathrm{mg} \cdot \mathrm{g}^{-1}\right)\end{array}$ & $\mathrm{k} 2\left(\mathrm{~g} \cdot \mathrm{mg}^{-1} \cdot \mathrm{min}^{-1}\right)$ & $\mathrm{h}\left(\mathrm{mg}^{\left.-\mathrm{g}^{-1} \cdot \mathrm{min}^{-1}\right)}\right.$ & Equação & $\mathrm{R}$ \\
\hline 25 & 0,370 & 22,22 & 3,042 & $\mathrm{y}=0,392 \mathrm{x}+0,3272$ & 0,999 \\
37 & 0,374 & 19,744 & 2,762 & $\mathrm{y}=0,3867 \mathrm{x}+0,3621$ & 0,9987 \\
50 & 0,302 & 8,890 & 0,811 & $\mathrm{y}=0,3879 \mathrm{x}+1,2334$ & 0,9915 \\
\hline
\end{tabular}

A água produzida real e o efluente sintético foram comparados, nas Figuras 3.4 e 3.5. Os experimentos conduzidos a uma temperatura fixa de $25^{\circ} \mathrm{C}$, mostraram a semelhança na modelagem cinética de ambos os efluentes. Novamente a equação de pseudo segunda ordem revelou-se um melhor ajuste quando comparado com a equação de pseudo- primeira ordem ( $\mathrm{R}$ próximo a 1$)$.

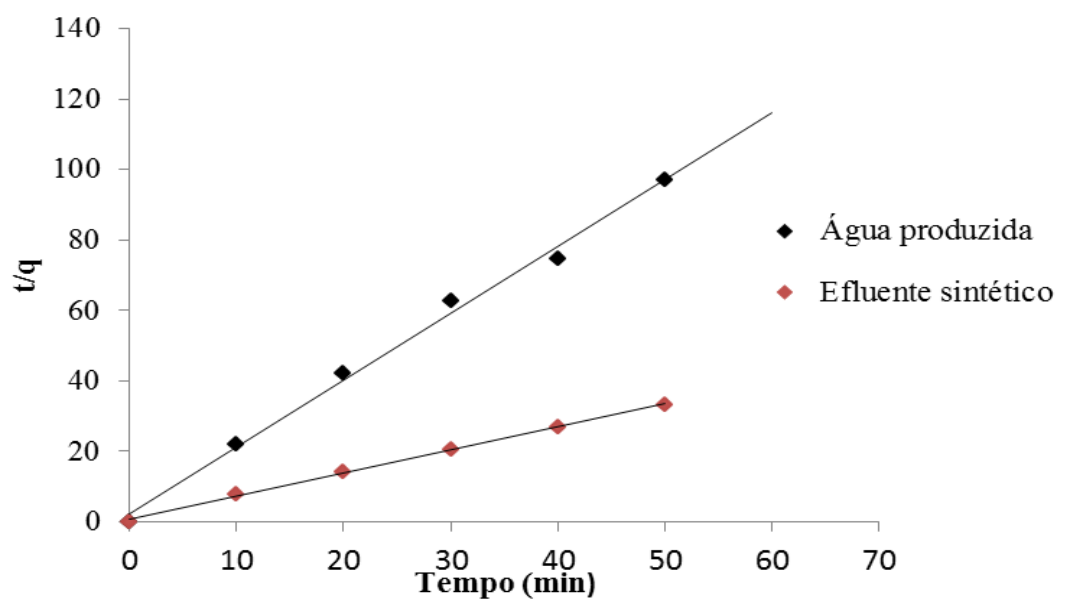

Figura 3.4 - Modelo pseudo $1^{\circ}$ ordem para a água produzida real e o efluente sintético a $\mathrm{T}=25^{\circ} \mathrm{C}$

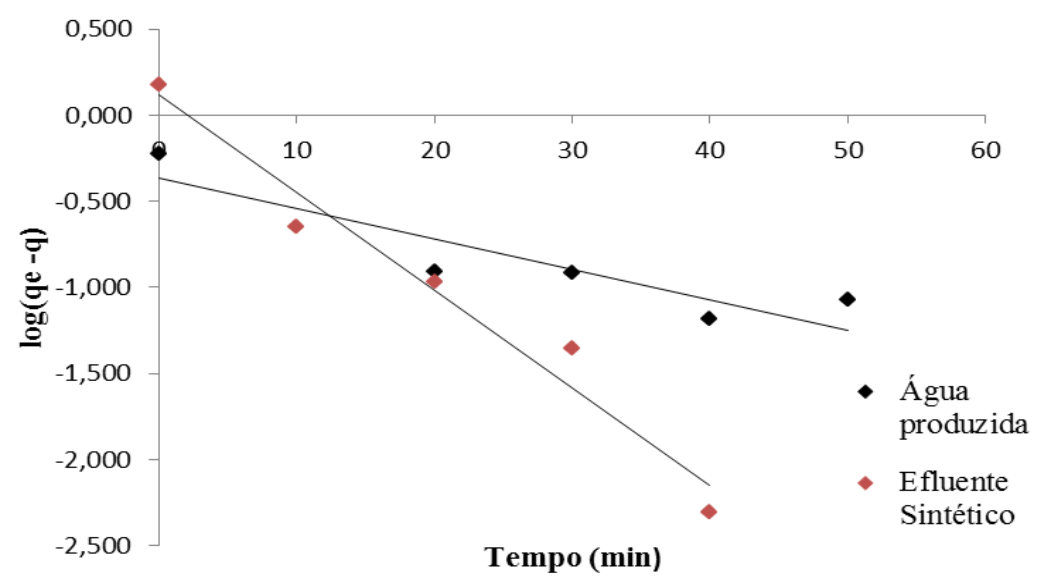

Figura 3.5 - Modelo pseudo - segunda ordem para água produzida real e o efluente sintético a $\mathrm{T}=25^{\circ}$ 


\subsection{Equilíbrio de adsorção}

Os dados experimentais das isotermas foram ajustados com as equações de Langmuir e Freundlich. Na Tabela 3.3, estão apresentados os parâmetros obtidos pelos ajustes. Ambos os modelos apresentaram bons ajustes, porém o modelo de Langmuir apresentou correlação melhor, indicando que a adsorção que irá prevalecer é em monocamada.

Tabela 3.3 - Parâmetros das isotermas de Freundlich e Langmuir para o efluente sintético a $\mathrm{T}=25^{\circ} \mathrm{C}$

\begin{tabular}{c|c|c|c}
\hline & $\begin{array}{c}\text { qmáx } \\
\mathrm{mg} / \mathrm{g}\end{array}$ & $\mathrm{Kd}$ & $\mathrm{R}$ \\
\hline Parâmetros de Langmuir & 0,405 & $-0,148$ & 0,988 \\
& & & \\
\hline Parâmetros de Freundlich & $-0,3622$ & $10^{2,96}$ & 0,955 \\
\hline
\end{tabular}

\section{CONCLUSÃO}

Os resultados mostraram a excelente capacidade de adsorção do carvão ativado na adsorção do carbono orgânico tanto no efluente sintético quanto na água produzida real. Apesar dos dois modelos cinéticos (primeira e segunda ordem), apresentarem ajustes o modelo pseudo segunda ordem se mostrou melhor para ambos os efluentes e para toda variação de temperatura $\left(25^{\circ} \mathrm{C}, 37^{\circ} \mathrm{C}\right.$ e $\left.50^{\circ} \mathrm{C}\right)$, no entanto a cinética para o efluente sintético foi mais eficiente nas temperaturas $25^{\circ} \mathrm{C}$ e $37^{\circ} \mathrm{C}$. Os modelos de isotermas também apresentaram bons ajustes para Langmuir e Freundlich, no entanto, Lagmuir prevaleceu indicando uma adsorção em monocamada.

\section{REFERÊNCIAS}

Alhamed, Y. A, Adsorption kinetics and performance of packed bed adsorber for phenol removal using activated carbono from dates' stones. Journal Hazardous Materials , v.170, p. 763 - 770, 2009.

Benkhedda, J; Jaubert, J, N; Barth. D; Perrin, L; Baily, M. Adsorption isotherms of $m$-xylene on activated carbon: measurements and correlation with different models, J.Chem.Thermodymamics, 32, 401-411, 2000.

Claudino, Andréia. Preparação de carvão ativado a partir de turfa e sua utilização na remoção de poluentes. Florianópolis: Universidade Federal de Santa Catarina, 2003. Dissertação (Mestrado).

Gomide, R. Operações Unitárias, Edição do autor, São Paulo, 1980.

Ho, Y. S. Review of second-order models for adsorption systems. Journal of Hazardous Materials, v. 136, p. 681-689, 2006. 
Ho, Y.S.; Mckay, G. Sorption of dye from aqueous solution by peat. Chemical Eng Journal, v. 70, p. 115-124, 1998.

Ho, Y.S.; Mckay, G. The kinetics of sorption of divalent metal ions onto sphagnum moss peat. Water Res., v. 34(3), p. 735-42, 2000.

Lagergren, S; Ksver. Vaterskapsakad. Handl, v.24 (1898) p.1.

Laszló, K; Podloscielny, P; Dabrowsk, A. Heterogeneity of activated carbons with different surface chemistry in adsorption of phenol from aqueous solutions. Applied Surface Science, v.252 p.5752 - 5762, 2005.

Lillo - Ródenas, M.A.; Fletcher, A.J.; Thomas, K.M.; Cazorla - Amorós, D.; Linares - Solano, A. Competitive adsorption of a benzene-toluene mixture on activated carbons at low concentration. Carbon, v. 441 p.455-1463, Janeiro,2006.

Lorenc - Grabowska. E; Gryglewicz. G; Diez, M. A. Kinectics and equilibrium study of phenol adsorption on nitrogen - enriched activated carbons. Fuel, v. 114, p. 235 - 243, 2009.

MCKAY, G. Use of adsorbents for removal of pollutants from wastewater. Florida: CRC Press, 186 p. 1996.

Mariano, J.B. Impactos ambientais do refine de petróleo. 1 ed. - Rio de Janeiro: Interciência, 2005.

Maretto, M; Blonchi, F; Vignola, R; Canepari, S; Baric, M; Lazzoni, R; Tagliabue, M; Papini, M, P. Microporaus and mesoporous materials for the treatment of wastwater produced by petrochimical activeties. Journal of cleaner production, v. 77, p. 22-34, 2014.

Neff, J; Sauer, T; Maciolek, N. Composition fate and effects of produced water discharges to nearshore marine Waters. Produced water. Plenum, New York, 1992.

Pei. J; Zhang, J, S. Determination of adsorption isotherm and diffusion coeficiente of toluene on actived carbono at low concentrations, v. 45, p. 66 -76. 2012.

Roostei, N.; Tezel, F.H.; Removal of phenol from aqueous solutions by adsorption. Journal of Environmental Management v.70 p.157-164, 2003.

Ruthven, D. M. Principles of adsorption and adsorption processes. New York: Wiley, 1984.

Veil, J. A; Puder, M. G; Elcock, D; Redweik, R. J. Jr. (2004), A White Paper Describing Produced Water from Production of Crude Oil Natural Gas, and Coal Bed Methane. U. S. Department of Energy - National Energy Technology Laboratory. Under Contract W-31-109-Eng-38.

Vegueria, S. F. J; Godoy, J. M; Miekeley, N. Environmental impact studies of barium and radium discharges by produced Waters from the "Bacia de Campos" Oil - field odffshore plataforms, Brazil of Environmental Radioactivicity.v. 62, p. 29 - 38, 2002. 